providing excellent cancer care. As a result, our multidisciplinary team looked at our current pathway and evaluated it against best practices, recent directives and programmes such as the Accelerate, Coordinate, and Evaluate (ACE) Programme. The ACE programme encourages the utilisation of Straight-toTest (STT) to improve time to diagnosis, and so we challenged ourselves to implement it effectively by appointing a dedicated 'Pathway Coordinator' (Band 6 Nurse).

The coordinator receives referrals, triages them with consultants, and then places them either into clinics or directly to investigations (STT). They also conduct a preprocedure assessment over telephone within 48-72 hours that helps confirm the patient's indication, fitness and willingness to have a definitive test. By collecting and comparing data on whether patients attend clinic or go directly to scope and how long it takes for them to receive treatment, before and after the new job role, we can accurately assess the new pathways' direct impact on patients receiving treatment.

Initial Results showed that nearly half of patients went straight to test, with an improved number of days between referral and scope and referral and diagnosis, indicating that adapting this patient centred approach through a targeted role effectively addresses some of the challenges that the $2 \mathrm{ww}$ pathway presents.

\section{DEVELOPING THE 'F3' PROGRAM AT ROYAL SURREY COUNTY HOSPITAL (RSCH)}

\section{Layla Brookfield. UK}

\subsection{6/leader-2019-FMLM.42}

Introduction As the downward trend in the number of Foundation Year 2 doctors progressing directly to speciality training continues, NHS trusts are left struggling with rota gaps and reliant on locums. The Royal Surrey County Hospital (RSCH) is no exception. This project aimed to understand why current F2s have decided to take time out and their aims for the coming year. This information then informs the development of an attractive Foundation Year 3 (F3) program that benefits both the trust and the doctor.

Method A list of current F2s was provided by the Medical Education department. A survey was sent using Survey Monkey. Responders were asked both multiple choice and ranking questions. These concerned: their plans for the coming year, what they are hoping to get out of the coming year, and what they would look for in a trust grade position. Results were analysed and graphically represented using Microsoft Excel.

Results 25 doctors responded to the survey. Five are going directly into training; the remaining 20 are taking a year out. There are several reasons for this but the most common was to improve work life balance with $70 \%$ citing this as a reason. Just over half also want to travel. More doctors are considering locum work over trust grade employment. Competitive pay and choice of speciality are the most important factors for doctors when considering trust grade appointment. Flexibility in contract length is also an important factor.

Conclusion Only 20\% of doctors in this cohort are going into speciality training. There are multifactorial reasons for this but most are seeking to improve work life balance. Many doctors are looking to take up service posts in the UK before making use of the greater flexibility to travel Doctors favour locuming over trust grade appointment but trust grades can be made more attractive by offering flexible contract lengths, competitive pay and choice of speciality.

\section{Leading Across Systems and Organisations}

\section{HOW TO STOP GUIDELINES FROM GATHERING DUST ON THE SHELF: EVIDENCE-BASED INTERVENTIONS}

\author{
${ }^{1,2}$ Aoife Molloy, ${ }^{2}$ Johannes Wolff. ${ }^{1}$ Imperial College London, UK; ${ }^{2}$ NHS England and NHS \\ Improvement, UK
}

10.1136/leader-2019-FMLM.43

The Evidence-Based Interventions Programme was established and developed as a joint enterprise between five national partners: the Academy of Medical Royal Colleges, NHS Clinical Commissioners, the National Institute for Health and Care Excellence as well as NHS England and NHS Improvement to lead national implementation of appropriate clinical intervention in an integrated health economy. The programme aims to ensure that procedures are offered appropriately in the NHS in England by using shared decision making, behavioural insights, consensus-seeking, collaboration with clinicians and commissioners, contract amendments, tariff changes and patient engagement. Lessons learned are wide ranging and emphasise the need for leadership at all levels of the health care system to inspire improvement:

1. We should ensure that procedures are offered appropriately in the NHS in England.

2. National implementation of initiatives is complex and requires diverse public and stakeholder engagement.

3. Creating nuanced messaging across diverse groups should not be shyed away from though it is challenging.

\section{$44 \quad$ IMPROVEMENT SCIENCE INFORMING CLINICAL STRATEGY}

FFPH Malti Varshney, Selina Robinson. London Clinical Networks and Senate, NHS England and Improvement (London), UK

\subsection{6/leader-2019-FMLM.44}

In 2015, the NHS, local councils, the Mayor and Public Health England set out their objectives for London's health and care system. National priorities were published in the NHS Long-Term Plan. London has a complex health and social care system serving 8.8 million population with high levels of inequality. A strategic partnership was formed identifying specific life outcomes and clinical priorities which will improve faster if the organisational partnerships works effectively at a 'once for London' level.

Initiatives by various organisations identified challenges relating to unwarranted variation in access, clinical practice and clinical outcomes. An effective delivery model was needed to coordinate efforts of all agencies to implement large scale change at system level. 
Interventions undertaken to develop pan-London clinical priorities

Development of London Clinical Network (LCN) Delivery Model using improvement science principles.

Development of weighted criteria for pan-London action

Framework developed on the role of the Regional Medical team

Clinical strategy event for senior professionals and clinical leaders to consider the issues and priorities which should form the basis of a renewed vision and strategy.

Using its Delivery model the LCN will be the primary delivery vehicle working alongside key stakeholders, 2019/20 delivery plans have been developed for priority areas. Improvements will be measured through reporting on indicators to Programme Boards through the Regional Medical Director.

The successful use of science to develop a strategy for action by bringing wider stakeholders together promoting a collaborative approach and galvanising commitment from the wider quality improvement architecture to work jointly to drive improvement.

System wide leadership brings local benefits, reduced duplication, enables shared learning and better use of resources. Trust and a commitment to collaborative working are required to bring about impactful change.

\section{IMPROVING CARDIOVASCULAR DISEASE (CVD) PREVENTION IN LONDON; A CO-ORDINATED APPROACH}

H Al-Omari, H Cutting, C Thomson. London Clinical Network, NHS England and Improvement, London, UK

\subsection{6/leader-2019-FMLM.45}

Issue Multiple agencies interested in improving Cardiovascular Disease (CVD) prevention were working in silos, resulting in repeated approaches to CCGs offering a variety of solutions in partnership with different organisations, causing confusion and limited outcomes.

Intervention London CCGs requested the Cardiac and Stroke Clinical Networks to develop a unified approach. The Networks engaged improvement organisations forming the London Cardiovascular Disease Prevention Partnership (CVDPP) to achieve this for Atrial Fibrillation, Hypertension and Familial Hypercholesterolaemia. Condition specific steering groups were formed to provide clinical, patient and system leadership and support delivery of improvement projects.

Strategy for improvement The CVDPP held workshops attended by numerous system partners to develop a London vision for CVD prevention, share best practice, include patients and discuss solutions to common barriers.

A RACI was used to clarify roles of organisations engaged in CVD prevention. Detailed plans for each condition are being developed with STPs using a logic model and menu of evidence-based interventions. Agreed detection and treatment targets for each condition to be achieved by 2023.

Impact

1. Increased detection and management of CVD risk factors;

2. Additional 400 lives saved each year by reducing heart attacks and strokes;
3. Empowering Londoners to take control of their circulatory health.

Lessons learnt Working collaboratively and innovatively across organisations with competing priorities brings complexities. Implementing large scale change across a complex and varied landscape requires patience, flexibility and time coupled with a clear aim.

Messages for others Working in a co-ordinated partnership minimises duplication and maximises utilisation of limited resources to achieve large scale change. Multiple and sometimes conflicting approaches to commissioners has been eliminated through partnership working.

\section{IMPROVING WEEKEND MEDICAL WARD COVER AT ROYAL SURREY COUNTY HOSPITAL}

Mengfei Lu, Kishan Moosai, Roselle Herring. Royal Surrey County Hospital, UK

\subsection{6/leader-2019-FMLM.46}

Introduction A 7-day NHS service is proven to achieve greatest benefits for patients as published by recent evidence based review. Despite the limitation of NHS resources, healthcare professionals should aim to provide the best quality of care to patients at all times.

Concerns about weekend ward cover, especially for medical patients on outlier wards, were raised through emails and verbal feedback from doctors and nursing teams. This project aims to improve clinical effectiveness for medical patients over the weekend.

Methods Qualitative data was collected through verbal and written feedbacks from wards, managers and medical teams.

A new poster with up-to-date bleep numbers for medical doctors over the weekend was designed, and a morning weekend handover was established together with collection of bleeps in a designated area. These changes were communicated to doctors, site managers, nursing and administrative staff and switchboard.

Results Numerous factors which were contributing to the problem have been identified:

1. Incorrect bleep numbers were recorded on the switchboard system

2. The use of bleeps were inconsistent amongst doctors

3. The ward staffs were unaware of the bleep numbers that were carried by the doctors

4. Uncertainty of which doctors are on-call

Conclusion Several implementations have been introduced as a result to improve communication between ward staffs and oncall doctors.

1. Designated buddying outlier wards for specific medical wards.

2. Designated bleeps numbers for doctors covering specific wards, all bleeps need to be returned to EAU office at the end of the shift.

3. Up-to-date on-call board on EAU

4. Laminated A4 medical on-call bleep sheet on every ward to guide ward staffs to bleep the accurate doctors.

5. Up-to-date list of bleeps with switchboard

6. Emails to all medical consultants, trainees, ward team and site managers with updates of the implementation. 